be emitted without altering the spin state; a similar approach has already been used for high-fidelity readout in other systems, such as trapped ions. A single-shot spin measurement - without the need for averaging over several measurements was not demonstrated as part of this work, but it seems to be within reach.

Taken together, these results ${ }^{3,4}$ seem to open many new possibilities with quantum dots. From a technical viewpoint, any optical control or detection scheme that is possible in atoms is now feasible for quantum dots, even those involving resonant excitation and collection of photons scattered at the same wavelength, or excitation powers far in excess of the saturation power. Some interesting problems remain, however. For example, is there any way to obtain in the same quantum dot both a 'cycling transition' (which Vamivakas et al. ${ }^{3}$ demonstrated to be optimal for readout), and ' $\Lambda$-type transitions'2, which are good for optical spin manipulation and the conversion of information stored in the stationary quantum-dot qubit to 'flying' photonic qubits? In any case, we expect to see rapid progress over the next few years in areas such as single-shot readout and stationaryto-flying-qubit conversion that will realize the promise of quantum dots as a system that is suitable for quantum-information applications and yet fully integrable into a microphotonic network.

Charles Santori is in the Information and Quantum Systems Laboratory (IQSL),
Hewlett-Packard Laboratories, 1501 Page Mill Road, Palo Alto, California 94304, USA.

e-mail: charles.santori@hp.com

Yoshihisa Yamamoto is in the E. L. Ginzton Laboratory, Stanford University, Stanford, California 94305-4088, USA.

e-mail:yyamamoto@stanford.edu

References

1. Xu, X. et al. Science 317, 929-932 (2007).

2. Press, D., Ladd, T. D., Zhang, B. \& Yamamoto, Y. Nature 456, 218-221 (2008)

3. Vamivakas, A. N., Zhao, Y., Lu, C.-Y. \& Atatüre, M. Nature Phys. 5, 198-202 (2009).

4. Flagg, E. B. et al. Nature Phys. 5, 203-207 (2009).

5. Mollow, B. R. Phys. Rev. 188, 1969-1975 (1969).

6. Wu, F. Y., Grove, R. E. \& Ezekiel, S. Phys. Rev. Lett. 35, 1426-1429 (1975).

7. Wrigge, G., Gerhardt, I., Hwang, J., Zumofen, G. \& Sandoghdar, V Nature Phys. 4, 60-66 (2008).

\title{
NUCLEAR MAGNETIC RESONANCE
}

\section{The benefits of travel}

Nuclear magnetic resonance (NMR) is widely exploited for imaging, particularly in medicine. Writing in Nature,

David Brunner and colleagues propose a subtle change to the NMR technique that could improve both the clarity of the images produced and the experience of a human patient during imaging

(Nature 457, 994-998; 2009).

Traditionally in NMR imaging a standing radio-frequency wave is created within the sample under study, causing the atoms' nuclear magnetization to 'wobble' (or nutate); the subsequent precession of the magnetization is then picked up by a probe and processed into images. Brunner et al., however, have found advantages to using a travelling, rather than a standing, radiofrequency wave.

The motivation comes from the electrodynamics of the situation: the magnetic field must exhibit curvature, and for a standing wave that means spatial variation in the magnitude of the field; for a travelling wave, however, the curvature can instead be accommodated in phase variation, which means that the magnitude of the field is more uniform, and therefore better for imaging.

Using a travelling wave also means that the NMR probe must be configured differently. In the standingwave case, the probe (and hence any potentially hazardous, strong electric field emanating from it) is positioned very close to the sample, or patient.

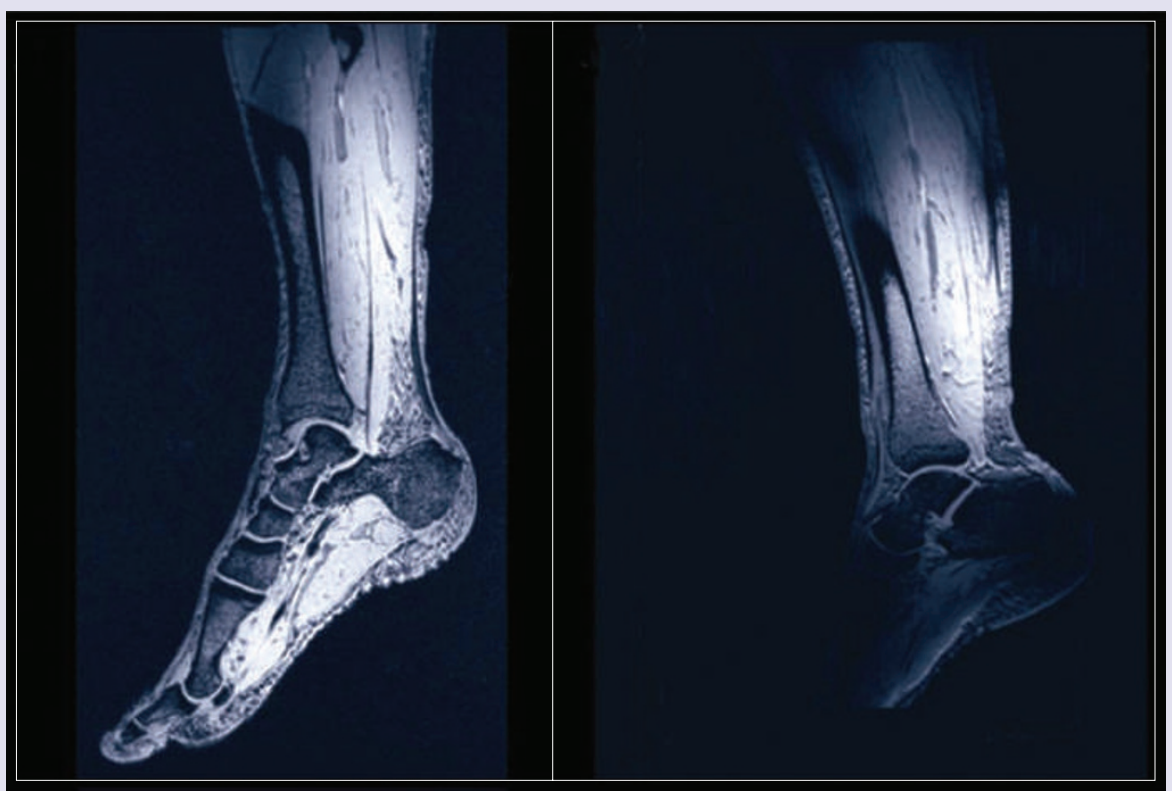

For travelling-wave NMR, however, the probe can instead be moved some distance away, because it couples to the propagating modes of the waveguide formed by the body of the scanner. This improves the performance of the probe, but it also frees up more space inside the scanner for the patient claustrophobia can often be a problem for patients undergoing scans.

In the lower-leg scans shown here, produced by Brunner et al., the benefit of imaging using a travelling wave (left) is clear, compared with the conventional approach (right). There are challenges to extending the travelling-wave technique to whole-body imaging - notably diffraction and attenuation at dielectric interfaces such as the shoulders. But the authors suggest that wave impedance matching (which they have already been able to demonstrate using bottles of mineral oil as imaging samples) should be an effective method for counteracting such effects. 